\title{
SOME NEW BRUNN-MINKOWSKI-TYPE INEQUALITIES IN CONVEX BODIES
}

\author{
ZHAO CHANG-JIAN, LENG GANGSONG, AND LOKENATH DEBNATH
}

Received 17 August 2004 and in revised form 10 December 2004

We establish some analogues of the Brunn-Minkowski inequalities on convex bodies and the Minkowski inequality and their inverse versions. As an application, we generalize and improve some interrelated results.

\section{Introduction}

The Brunn-Minkowski inequality theory plays an important role in a number of mathematical disciplines such as measure theory, crystallography, optimal control theory, functional analysis, and geometric convexity. It has many useful applications in combinatorics, stochastic geometry, and mathematical economics.

In recent years, several authors including Ball $[1,2,3]$, Bourgain and Lindenstrauss [7], Gardner [8, 9, 10], Schneider [25], Lutwak [12, 13, 14, 15, 16, 17, 18, 19, 20, 21, 22, 23], have given considerable attention to the Brunn-Minkowski inequality theory and its various generalizations. The purpose of this paper is to establish some new-type BrunnMinkowski inequalities and Minkowski inequalities and their inverse versions. As an application, we generalize and improve some interrelated results.

Notation and preliminary works are given in Section 2.

In Section 3.1, some new inequalities of width-integrals, with equality conditions for convex bodies and their inverse versions are established. And we also establish some similar inequalities for projection bodies. This work generalizes a result which was given in [13]. Most importantly, we find the Brunn-Minkowski inequality for polars of mixed projection bodies in the section.

Generalizations and inverse versions of the dual Minkowski inequality and BrunnMinkowski inequalities, with inequality conditions, for the radial Minkowski linear combination are presented in Section 3.2. These results generalize some results which were given by Lutwak $[12,17]$.

A general dual Brunn-Minkowski inequality, with equality conditions for radial Blaschke linear combination and its inverse version were given in Section 3.3. A special case of the result is just a new inequality, which was given by Lutwak [17]. A dual Brunn-Minkowski inequality for the harmonic Blaschke linear combination and its inverse version, with equality conditions, were established in Section 3.4.

Copyright (C) 2005 Hindawi Publishing Corporation

International Journal of Mathematics and Mathematical Sciences 2005:6 (2005) 895-915

DOI: 10.1155/IJMMS.2005.895 
A general Minkowski inequality and Brunn-Minkowski inequalities of affine surface area for the Blaschke linear combination and their inverse versions (with equality conditions) for convex bodies are presented in Section 3.5. In this section, we also establish some similar inequalities for the harmonic linear combination. Our work generalizes some results of Lutwak $[16,18]$.

Finally, in Section 3.6, we give the dual Brunn-Minkowski inequality of affine surface area and its inverse version, with equality conditions for the Lutwak linear combination.

\section{Notations and preliminary ideas}

The setting for this paper is $n$-dimensional Euclidean space $\mathbb{R}^{n}(n>2)$. Let $\mathbb{C}^{n}$ denote the set of nonempty convex figures (compact, convex subsets) and let $\mathcal{K}^{n}$ denote the subset of $\mathbb{C}^{n}$ consisting of all convex bodies (compact, convex subsets with nonempty interiors) in $\mathbb{R}^{n}$, and if $p \in \mathscr{K}^{n}$, let $\mathscr{K}_{p}^{n}$ denote the subset of $\mathscr{K}^{n}$ that contains the centered (centrally symmetric with respect to $p$ ) bodies. We reserve the letter $u$ for unit vectors, and the letter $B$ is reserved for the unit ball centered at the origin. The surface of $B$ is $S^{n-1}$. For $u \in S^{n-1}$, let $E_{u}$ denote the hyperplane through the origin, that is orthogonal to $u$. We will use $K^{u}$ to denote the image of $K$ under an orthogonal projection onto the hyperplane $E_{u}$.

2.1. Mixed volumes and radial functions. We use $V(K)$ for the $n$-dimensional volume of convex body $K$. Let $h(K, \cdot): S^{n-1} \rightarrow \mathbb{R}$ denote the support function of $K \in \mathscr{K}^{n}$; that is,

$$
h(K, u)=\operatorname{Max}\{u \cdot x: x \in K\}, \quad u \in S^{n-1},
$$

where $u \cdot x$ denotes the usual inner product $u$ and $x$ in $\mathbb{R}^{n}$.

Let $\delta$ denote the Hausdorff metric on $\mathcal{Y}^{n}$; that is, for $K, L \in \mathscr{Y}^{n}$,

$$
\delta(K, L)=\left|h_{K}-h_{L}\right|_{\infty}
$$

where $|\cdot|_{\infty}$ denotes the sup-norm on the space of continuous functions $C\left(S^{n-1}\right)$.

For a convex body $K$ and a nonnegative scalar $\lambda, \lambda K$ is used to denote $\{\lambda x: x \in K\}$. For $K_{i} \in \mathscr{K}^{n}, \lambda_{i} \geq 0(i=1,2, \ldots, r)$, the Minkowski linear combination $\sum_{i=1}^{r} \lambda_{i} K_{i} \in \mathscr{K}^{n}$ is defined by

$$
\sum_{i=1}^{r} \lambda_{i} K_{i}=\left\{\sum_{i=1}^{r} \lambda_{i} x_{i} \in K^{n}: x_{i} \in K_{i}\right\} .
$$

It is trivial to verify that

$$
h\left(\sum_{i=1}^{r} \lambda_{i} K_{i}, \cdot\right)=\sum_{i=1}^{r} \lambda_{i} h\left(K_{i}, \cdot\right) .
$$

If $K_{i} \in \mathscr{K}^{n}(i=1,2, \ldots, r)$ and $\lambda_{i}(i=1,2, \ldots, r)$ are nonnegative real numbers, then of fundamental importance is the fact that the volume of $\sum_{i=1}^{r} \lambda_{i} K_{i}$ is a homogeneous 
polynomial in $\lambda_{i}$ given by Schneider [25]

$$
V\left(\sum_{i=1}^{r} \lambda_{i} K_{i}\right)=\sum_{i_{1}, \ldots, i_{n}} \lambda_{i_{1}} \cdots \lambda_{i_{n}} V_{i_{1}, \ldots, i_{n}}
$$

where the sum is taken over all $n$-tuples $\left(i_{1}, \ldots, i_{n}\right)$ of positive integers not exceeding $r$. The coefficient $V_{i_{1}, \ldots, i_{n}}$ depends only on the bodies $K_{i_{1}}, \ldots, K_{i_{n}}$, and is uniquely determined by (2.5), it is called the mixed volume of $K_{i}, \ldots, K_{i_{n}}$, and is written as $V\left(K_{i_{1}}, \ldots, K_{i_{n}}\right)$. Let $K_{1}=\cdots=K_{n-i}=K$ and let $K_{n-i+1}=\cdots=K_{n}=L$, then the mixed volume $V\left(K_{1}, \ldots, K_{n}\right)$ is usually written as $V_{i}(K, L)$. If $L=B$, then $V_{i}(K, B)$ is the $i$ th projection measure (Quermassintegral) of $K$ and is written as $W_{i}(K)$. With this notation, $W_{0}=V(K)$, while $n W_{1}(K)$ is the surface area of $K, S(K)$.

Associated with a compact subset $K$ of $\mathbb{R}^{n}$, which is star-shaped with respect to the origin, its radial function $\rho(K, \cdot): S^{n-1} \rightarrow \mathbb{R}$ is defined for $u \in S^{n-1}$ by

$$
\rho(K, u)=\operatorname{Max}\{\lambda \geq 0: \lambda u \in K\} .
$$

If $\rho(K, \cdot)$ is positive and continuous, $K$ will be called a star body. Let $\varphi^{n}$ denote the set of star bodies in $\mathbb{R}^{n}$ and let $\varphi_{c}^{n}$ denote the subset of $\varphi^{n}$ that contains the centered bodies (centrally symmetric with respect to $c$ ). If, for $K \in \varphi^{n}, \rho(K, \cdot)$ is even (assumes the same values at antipodal points), then $K$ is called centered.

The radial Hausdorff distance $\tilde{\delta}(K, L)$ of $K, L \in \varphi^{n}$, is defined by Lutwak [18] as

$$
\tilde{\delta}(K, L)=\left|\rho_{K}-\rho_{L}\right|_{\infty} .
$$

It will be helpful to extend the domain of the radial function from $S^{n-1}$ to $\mathbb{R}^{n}-\{0\}$. For $K \in \varphi^{n}$, and $x \in \mathbb{R}^{n}-\{0\}$, define $\rho(K, x)$ by

$$
\rho(K, x)=\operatorname{Max}\{\lambda>0: \lambda x \in K\} .
$$

The polar coordinate formula for the volume in $\mathbb{R}^{n}$ is

$$
V(K)=\frac{1}{n} \int_{S^{n-1}} \rho(K, u)^{n} d S(u) .
$$

2.2. Width-integrals of convex bodies. For $u \in S^{n-1}, b(K, u)$ is defined to be half the width of $K$ in the direction $u$. Two convex bodies $K$ and $L$ are said to have similar width if there exists a constant $\lambda>0$ such that $b(K, u)=\lambda b(L, u)$ for all $u \in S^{n-1}$. For $K \in \mathscr{K}^{n}$ and $p \in \operatorname{int} K$, we use $K^{p}$ to denote the polar reciprocal of $K$ with respect to the unit sphere centered at $p$. Width-integrals were first considered by Blaschke $[4,5]$. The width-integral of index $i$ is defined by

$$
B_{i}(K)=\frac{1}{n} \int_{S^{n-1}} b(K, u)^{n-i} d S(u) \quad \text { for } K \in \mathscr{K}^{n}, i \in \mathbb{R},
$$

where $d S$ is the $(n-1)$-dimensional volume element on $S^{n-1}$. 
The width-integral of index $i$ is a map

$$
B_{i}: \mathscr{K}^{n} \longrightarrow \mathbb{R}
$$

It is positive, continuous, homogeneous of degree $n-i$, and invariant under motion. In addition, for $i \leq n$, it is also bounded and monotone under set inclusion.

The following results due to Lutwak [13] will be used later:

$$
\begin{gathered}
b(K+L, u)=b(K, u)+b(L, u), \\
B_{2 n}(K) \leq V\left(K^{p}\right),
\end{gathered}
$$

with equality if and only if $K$ is symmetric with respect to $p$.

2.3. The radial Minkowski linear combination and dual mixed volumes. We define a vector addition on $\mathbb{R}^{n}$, which we call radial addition as follows: if $x_{1}, \ldots, x_{r} \in \mathbb{R}^{n}$, then $x_{1} \tilde{+} \cdots \tilde{+} x_{r}$ is defined to be the usual vector sum of $x_{1}, \ldots, x_{r}$ provided $x_{1}, \ldots, x_{r}$ all lie in 1 -dimensional subspace of $\mathbb{R}^{n}$, and as the zero vector otherwise.

If $K_{1}, \ldots, K_{r} \in \varphi^{n}$ and $\lambda_{1}, \ldots, \lambda_{r} \in \mathbb{R}$, then the radial Minkowski linear combination $\lambda_{1} K_{1} \tilde{+} \cdots \tilde{+} \lambda_{r} K_{r}$ is defined by Lutwak [17] as

$$
\lambda_{1} K_{1} \tilde{+} \cdots \tilde{+} \lambda_{r} K_{r}=\left\{\lambda_{1} x_{1} \tilde{+} \cdots \tilde{+} \lambda_{r} x_{r}: x_{i} \in K_{i}\right\} .
$$

It has the following important property: for $K, L \in \varphi^{n}$ and $\lambda, \mu \geq 0$,

$$
\rho(\lambda K \tilde{+} \mu L, \cdot)=\lambda \rho(K, \cdot)+\mu \rho(L, \cdot) .
$$

For $K_{1}, \ldots, K_{r} \in \varphi^{n}$ and $\lambda_{1}, \ldots, \lambda_{r} \geq 0$, the volume of the radial Minkowski linear combination $\lambda_{1} K_{1} \tilde{+} \cdots \tilde{+} \lambda_{r} K_{r}$ is a homogeneous $n$ th-degree polynomial in the $\lambda_{i}$,

$$
V\left(\lambda_{1} K_{1} \tilde{+} \cdots \tilde{+} \lambda_{r} K_{r}\right)=\sum \tilde{V}_{i_{1}, \ldots, i_{n}} \lambda_{i_{1}} \cdots \lambda_{i_{n}}
$$

where the sum is taken over all $n$-tuples $\left(i_{1}, \ldots, i_{n}\right)$ whose entries are positive integers not exceeding $r$. If we require the coefficients of the polynomial in (2.16) to be symmetric in their argument, then they are uniquely determined. The coefficient $\widetilde{V}_{i_{1}, \ldots, i_{n}}$ is nonnegative and depends only on the bodies $K_{i_{1}}, \ldots, K_{i_{n}}$. It is written as $\tilde{V}\left(K_{i_{1}}, \ldots, K_{i_{n}}\right)$ and is called the dual mixed volume of $K_{i_{1}}, \ldots, K_{i_{n}}$. If $K_{1}=\cdots=K_{n-i}=K, K_{n-i+1}=\cdots=K_{n}=L$, the dual mixed volumes $\widetilde{V}_{i}(K, B)$ is written as $\widetilde{W}_{i}(K)$.

For $K_{i} \in \varphi^{n}$, the dual mixed volumes are defined first by Lutwak [18] as

$$
\tilde{V}\left(K_{1}, \ldots, K_{n}\right)=\frac{1}{n} \int_{S_{n-1}} \rho\left(K_{1}, u\right) \cdots \rho\left(K_{n}, u\right) d S(u) .
$$

For $K, L \in \varphi^{n}$ and $i \in R$, the $i$ th dual mixed volume of $K$ and $L, \tilde{V}_{i}(K, L)$ is defined by

$$
\tilde{V}_{i}(K, L)=\frac{1}{n} \int_{S^{n-1}} \rho(K, u)^{n-i} \rho(L, u)^{i} d S(u) .
$$


From (2.18) and in view of $h(B, u)=1$, if $K \in \mathscr{Y}^{n}, i \in \mathbb{R}$,

$$
\tilde{W}_{i}(K)=\frac{1}{n} \int_{S^{n-1}} \rho(K, u)^{n-i} d S(u)
$$

2.4. The radial Blaschke linear combination. Lutwak [17] defined the radial Blaschke linear combination. If $K, L \in \varphi^{n}$ and $\lambda, \mu \geq 0$, then $\lambda \cdot K \breve{+} \mu \cdot L$, as the star body whose radial function is given by

$$
\rho(\lambda \cdot K \breve{+} \mu \cdot L, \cdot)^{n-1}=\lambda \rho(K, \cdot)^{n-1}+\mu \rho(L, \cdot)^{n-1} .
$$

We will call the addition and scalar multiplication the radial Blaschke addition and scalar multiplication. We note that for convex bodies, Blaschke scalar multiplication and the radial Blaschke scalar multiplication agree.

2.5. The harmonic Blaschke linear combination. A new addition called the harmonic Blaschke addition is defined by Lutwak [18]. Suppose $K, L \in \varphi^{n}$, and $\lambda, \mu \geq 0$ (not both zero). To define the harmonic Blaschke linear combination $\lambda K \hat{+} \mu L$, first define $\xi>0$ by

$$
\xi^{1 /(n+1)}=\frac{1}{n} \int_{S^{n-1}}\left[\lambda V(K)^{-1} \rho(K, u)^{n+1}+\mu V(L)^{-1} \rho(L, u)^{n+1}\right]^{n /(n+1)} d S(u) .
$$

The body $\lambda K \hat{+} \mu L \in \varphi^{n}$ is defined as the body whose radial function is given by Lutwak [18] in the form

$$
\xi^{-1} \rho(\lambda K \hat{+} \mu L, \cdot)^{n+1}=\lambda V(K)^{-1} \rho(K, \cdot)^{n+1}+\mu V(L)^{-1} \rho(L, \cdot)^{n+1}
$$

From this definition and (2.9), it follows immediately that $\xi=V(\lambda K \hat{+} \mu l)$, and hence

$$
V(\lambda K \hat{+} \mu L)^{-1} \rho(\lambda K \hat{+} \mu L, \cdot)^{n+1}=\lambda V(K)^{-1} \rho(K, \cdot)^{n+1}+\mu V(L)^{-1} \rho(L, \cdot)^{n+1} .
$$

2.6. Curvature functions and affine surface area. A convex body $K$ is said to have a positive continuous curvature function (see Lutwak [18]),

$$
f(K, \cdot): S^{n-1} \longrightarrow[0, \infty)
$$

for each $L \in \mathbb{C}^{n}$, the mixed volume $V_{1}(K, L)$ has the integral representation

$$
V_{1}(K, L)=\frac{1}{n} \int_{S^{n-1}} f(K, u) h(L, u) d S(u) .
$$

The subset of $\mathscr{K}^{n}$ consisting of bodies which have a positive continuous curvature function will be denoted by $\kappa^{n}$.

The following result is true (see Lutwak [16]): for $K \in \kappa^{n}$,

$$
\int_{S^{n-1}} u f(K, u) d S(u)=0
$$


900 Some new Brunn-Minkowski-type inequalities in convex bodies

Suppose $K, L \in \kappa^{n}$ and $\lambda, \mu \geq 0$ (not both zero). From (2.26), it follows that the function $\lambda f(K, \cdot)+\mu f(L, \cdot)$ satisfies the hypothesis of Minkowski's existence theorem (see Bonnesen and Fenchel [6]). The solution of the Minkowski problem for this function is denoted by $\lambda \cdot K \ddot{+} \mu \cdot L$; that is,

$$
f(\lambda \cdot K \ddot{+} \mu \cdot L, \cdot)=\lambda f(K, \cdot)+\mu f(L, \cdot),
$$

where the linear combination $\lambda K \ddot{+} \mu L$ is called the Blaschke linear combination.

The relationship between the Blaschke and Minkowski scalar multiplications is given by

$$
\lambda \cdot K=\lambda^{1 /(n-1)} K
$$

The affine surface area of $K \in \kappa^{n}, \Omega(K)$, is defined by

$$
\Omega(K)=\int_{S^{n-1}} f(K, u)^{n /(n+1)} d S(u) .
$$

It is well known that this functional is invariant under unimodular affine transformations. For $K, L \in \kappa^{n}$, and $i \in \mathbb{R}$, the $i$ th-mixed affine surface area of $K$ and $L, \Omega_{i}(K, L)$, was defined by Lutwak [18] as

$$
\Omega_{i}(K, L)=\int_{S^{n-1}} f(K, u)^{(n-i) /(n+1)} f(L, u)^{i /(n+1)} d S(u)
$$

Now, we define the $i$ th affine area of $K \in \kappa^{n}, \Omega_{i}(K)$, to be $\Omega_{i}(K, B)$, since $f(B, \cdot)=1$, we have

$$
\Omega_{i}(K)=\int_{S^{n-1}} f(K, u)^{(n-i) /(n+1)} d S(u), \quad i \in \mathbb{R} .
$$

Lutwak [17] also defined a mapping

$$
\Lambda: \varphi_{c}^{n} \longrightarrow \kappa_{c}^{n}
$$

and pointed out that $\Lambda$ transforms the harmonic Blaschke linear combination into the Blaschke linear combinations, that is, if $K, L \in \varphi_{c}^{n}$ and $\lambda, \mu \geq 0$, then

$$
\Lambda(\lambda K \hat{+} \mu L)=\lambda \cdot \Lambda K \ddot{+} \mu \cdot \Lambda L
$$

2.7. Projection bodies and the Lutwak linear combination. The projection body, $\Pi K$, of a convex body $K \in \mathcal{Y}^{n}$, is defined as the convex body whose support function is given by

$$
h(\Pi K, u)=v\left(K^{u}\right), \quad u \in S^{n-1} .
$$


It can be shown that (see Lutwak [17]) for $K, L, M \in \mathscr{Y}^{n}$,

$$
\Pi_{1}(M, K+L)=\Pi_{1}(M, K)+\Pi_{1}(M, L)
$$

where $\Pi_{1}(M, K)=\Pi(\underbrace{M, \ldots, M}_{n-2}, K)$.

The class $v^{n}$ is defined as follows:

$$
v^{n}=\left\{K \in \kappa^{n}: f(K, \cdot)^{-1 /(n+1)}=h(Q, \cdot)\right\}
$$

for some $Q \in \mathscr{K}^{n}$. The class $v^{n}$ has been extensively investigated by Petty [24].

Lutwak [18] again defined a new linear combination $\lambda \circ K \breve{+} \mu \circ L \in v^{n}$ (in this paper it is called the Lutwak linear combination). For centrally symmetric $K, L \in v^{n}$, we have

$$
f(K \check{+} L, \cdot)^{-1 /(n+1)}=f(K, \cdot)^{-1 /(n+1)}+f(L, \cdot)^{-1 /(n+1)} .
$$

\section{Main results}

\subsection{Inequalities for width-integrals}

(1) The dual Brunn-Minkowski inequality for width-integrals of convex bodies.

For the projection measures of the Minkowski sum $K+L$ of two convex bodies $K$ and $L$, we have the following inequality: if $K, L \in \mathscr{K}^{n}, i<n-1$,

$$
W_{i}(K+L)^{1 /(n-i)} \geq W_{i}(K)^{1 /(n-i)}+W_{i}(L)^{1 /(n-i)}
$$

with equality if and only if $K$ and $L$ are homothetic.

Similarly, for the width-integrals of the Minkowski sum of two convex bodies, Lutwak [13] established the following result: if $K, L \in \mathscr{K}^{n}, i<n-1$,

$$
B_{i}(K+L)^{1 /(n-i)} \leq B_{i}(K)^{1 /(n-i)}+B_{i}(L)^{1 /(n-i)}
$$

with equality if and only if $K$ and $L$ have similar widths.

Inequality (3.2) is called the dual Brunn-Minkowski inequality.

In fact, the inverse version of (3.2) also exists. If $K, L \in \mathscr{K}^{n}, i>n$, or $n-1<i<n$, then

$$
B_{i}(K+L)^{1 /(n-i)} \geq B_{i}(K)^{1 /(n-i)}+B_{i}(L)^{1 /(n-i)},
$$

with equality if and only if $K$ and $L$ have similar widths.

In addition, when $i<n-1$, we also obtain that

$$
B_{i}(K)+B_{i}(L) \leq B_{i}(K+L) .
$$

These inequalities are contained in the following theorem. 
902 Some new Brunn-Minkowski-type inequalities in convex bodies

Theorem 3.1. If $K_{j}(j=1, \ldots, m) \in \mathcal{M}^{n}$, then for $i<n-1$

$$
\left(\sum_{j=1}^{m} B_{i}\left(K_{j}\right)\right)^{1 /(n-i)} \leq B_{i}\left(\sum_{j=1}^{m} K_{j}\right)^{1 /(n-i)} \leq \sum_{j=1}^{m}\left(B_{i}\left(K_{j}\right)^{1 /(n-i)}\right) .
$$

The right equality of (3.5) holds if and only if $K_{j}$ have similar widths; the left equality of (3.5) holds if and only if, for almost all $u$, all but one of $b\left(K_{j}, u\right)$ are zero.

For $i>n$ or $n-1<i<n$,

$$
B_{i}\left(\sum_{j=1}^{m} K_{j}\right)^{1 /(n-i)} \geq \sum_{j=1}^{m}\left(B_{i}\left(K_{j}\right)^{1 /(n-i)}\right)
$$

with equality if and only if $K_{j}$ have similar widths.

To prove (3.5), we use (2.10), (2.12), and let $p=n-i$ and $f_{j}(u)=f\left(K_{j}, u\right)$ in the Minkowski inequality for integrals (see Hardy et al. [11], pages 147-148). Similarly, we can also prove (3.6).

In (3.6), let $i=2 n$; we have the following corollary.

Corollary 3.2. If $K, L \in \mathscr{Y}^{n}$, then

$$
B_{2 n}(K+L)^{-1 / n} \geq B_{2 n}(K)^{-1 / n}+B_{2 n}(L)^{-1 / n}
$$

with equality if and only if $K$ and $L$ have similar widths.

Taking $i=0$ to (3.5), we get the following corollary.

Corollary 3.3. If $K_{j} \in \mathscr{K}^{n}(j=1, \ldots, m)$, then

$$
B\left(K_{1}+\cdots+K_{m}\right)^{1 / n} \geq B\left(K_{1}\right)^{1 / n}+\cdots+B\left(K_{m}\right)^{1 / n}
$$

with equality if and only if $K_{j}$ have similar widths.

Taking $m=2$ to (3.8), inequality (3.8) reduces to (3.2).

(2) The Brunn-Minkowski inequality for width-integrals of projection bodies.

The following inequalities for width-integrals of the projection body will be established: if $K, L, M \in \mathscr{K}^{n}$, then, for $i<n-1$,

$$
B_{i}\left(\Pi_{1}(M, K+L)\right)^{1 /(n-i)} \leq B_{i}\left(\Pi_{1}(M, K)\right)^{1 /(n-i)}+B_{i}\left(\Pi_{1}(M, L)\right)^{1 /(n-i)}
$$

with equality if and only if $\Pi_{1}(M, K)$ and $\Pi_{1}(M, L)$ have similar widths.

For $i>n$ or $n-1<i<n$,

$$
B_{i}\left(\Pi_{1}(M, K+L)\right)^{1 /(n-i)} \geq B_{i}\left(\Pi_{1}(M, K)\right)^{1 /(n-i)}+B_{i}\left(\Pi_{1}(M, L)\right)^{1 /(n-i)}
$$

with equality if and only if $\Pi_{1}(M, K)$ and $\Pi_{1}(M, L)$ have similar widths.

These two inequalities are contained in the following theorem. 
Theorem 3.4. If $K_{j}(j=1, \ldots, m) \in \mathscr{K}^{n}$, then, for $i<n-1$,

$$
\left(\sum_{j=1}^{m} B_{i}\left(\Pi_{1}\left(M, K_{j}\right)\right)\right)^{1 /(n-i)} \leq B_{i}\left(\Pi_{1}\left(M, \sum_{j=1}^{m} K_{j}\right)\right)^{1 /(n-i)} \leq \sum_{j=1}^{m} B_{i}\left(\Pi_{1}\left(M, K_{j}\right)\right)^{1 /(n-i)} .
$$

The right equality of (3.11) holds if and only if $\Pi_{1}\left(M, K_{j}\right)(j=1, \ldots, m)$ have similar widths; the left equality of (3.11) holds if and only if, for almost all $u$, all but one of $b\left(\Pi_{1}((M\right.$, $\left.\left.\left.K_{j}\right), u\right)\right)(j=1, \ldots, m)$ are zero.

When $i>n$ or $n-1<i<n$,

$$
B_{i}\left(\Pi_{1}\left(M, \sum_{j=1}^{m} K_{j}\right)\right)^{1 /(n-i)} \geq \sum_{j=1}^{m} B_{i}\left(\Pi_{1}\left(M, K_{j}\right)\right)^{1 /(n-i)}
$$

with equality if and only if $\Pi_{1}\left(M, K_{j}\right)(j=1, \ldots, m)$ have similar widths.

Proof. First, we give the proof of (3.11).

From (2.10), (2.12), (2.35), and in view of the Minkowski inequality for integrals, we obtain

$$
\begin{aligned}
B_{i}\left(\Pi_{1}\left(M, \sum_{j=1}^{m} K_{j}\right)\right)^{1 /(n-i)} & =\left(\frac{1}{n} \int_{S^{n-1}} b\left(\Pi_{1}\left(M, \sum_{j=1}^{m} K_{j}\right), u\right)^{n-i} d S(u)\right)^{1 /(n-i)} \\
& =\left(\frac{1}{n} \int_{S^{n-1}} b\left(\sum_{j=1}^{m} \Pi_{1}\left(M, K_{j}\right), u\right)^{n-i} d S(u)\right)^{1 /(n-i)} \\
& =\left(\frac{1}{n} \int_{S^{n-1}}\left(\sum_{j=1}^{m} b\left(\Pi_{1}\left(M, K_{j}\right), u\right)\right)^{n-i} d S(u)\right)^{1 /(n-i)} \\
& \leq \sum_{j=1}^{m}\left(\frac{1}{n} \int_{S^{n-1}} b\left(\Pi_{1}\left(M, K_{j}\right), u\right)^{n-i} d S(u)\right)^{1 /(n-i)} \\
& =\sum_{j=1}^{m} B_{i}\left(\Pi_{1}\left(M, K_{j}\right)\right)^{1 /(n-i)} .
\end{aligned}
$$

On the other hand, we have

$$
\begin{aligned}
B_{i}\left(\Pi_{1}\left(M, \sum_{j=1}^{m} K_{j}\right)\right) & =\frac{1}{n} \int_{S^{n-1}}\left(\sum_{j=1}^{m} b\left(\Pi_{1}\left(M, K_{j}\right), u\right)\right)^{n-i} d S(u) \\
& \geq \sum_{j=1}^{m} \int_{S^{n-1}} b\left(\Pi_{1}\left(M, K_{j}\right), u\right)^{n-i} d S(u) \\
& =\sum_{j=1}^{m} B_{i}\left(\Pi_{1}\left(M, K_{j}\right)\right) .
\end{aligned}
$$

The proof of (3.11) is complete. 
904 Some new Brunn-Minkowski-type inequalities in convex bodies

Similarly, we also can prove (3.12).

Putting $i=2 n$ and $m=2$ to (3.12), we obtain the following corollary.

Corollary 3.5. If $K, L, M \in \mathscr{M}^{n}$, then

$$
B_{2 n}\left(\Pi_{1}(M,(K+L))\right)^{-1 / n} \geq B_{2 n}\left(\Pi_{1}(M, K)\right)^{-1 / n}+B_{2 n}\left(\Pi_{1}(M, L)\right)^{-1 / n}
$$

with equality if and only if $\Pi_{1}(M, K)$ and $\Pi_{1}(M, L)$ have similar widths.

From (3.15), (2.13), and in view of the centered projection body (centrally symmetric with respect to origin), we obtain the following corollary.

Corollary 3.6. If $K, L, M \in \mathcal{Y}^{n}$, then

$$
V\left(\Pi_{1}^{*}(M, K+L)\right)^{-1 / n} \geq V\left(\Pi_{1}^{*}(M, K)^{-1 / n}\right)+V\left(\Pi_{1}^{*}(M, L)^{-1 / n}\right)
$$

with equality if and only if $\Pi_{1}(M, K)$ and $\Pi_{1}(M, L)$ have similar widths.

This is just the Brunn-Minkowski inequality of polars of mixed projection bodies. This is a new result.

3.2. The dual Minkowski inequality and the Brunn-Minkowski inequality for the radial Minkowski linear combination. The dual Minkowski inequality and the AleksandrovFenchel inequality have been established by Lutwak [12]. These results can be stated, respectively, as follows.

If $K, L \in \mathscr{Y}^{n}$, then

$$
\tilde{V}_{1}(K, L)^{n} \leq V(K)^{n-1} V(L)
$$

with equality if and only if $K$ is a dilation of $L$.

If $K_{i} \in \mathscr{Y}^{n}$, then

$$
\tilde{V}\left(K_{1}, \ldots, K_{n}\right)^{m} \leq \prod_{i=0}^{m-1} \tilde{V}\left(K_{1}, \ldots, K_{n-m}, K_{n-i}, \ldots, K_{n-i}\right)
$$

with equality if and only if $K_{n-m+1}, K_{n-m+2}, \ldots, K_{n}$ are all dilations of each other.

In fact, the Brunn-Minkowski inequality can also be stated as (see Gardner [10], page 374) as follows.

If $K, L \in \mathscr{Y}^{n}$, then

$$
V(K \tilde{+} L)^{1 / n} \leq V(K)^{1 / n}+V(L)^{1 / n}
$$

with equality if and only if $K$ is a dilation of $L$.

The following general version of the dual Brunn-Minkowski inequality can be established. 
If $K, L \in \mathscr{K}^{n}, i<n-1$, then

$$
\tilde{W}_{i}(K \tilde{+} L)^{1 /(n-i)} \leq \tilde{W}_{i}(K)^{1 /(n-i)}+\tilde{W}_{i}(L)^{1 /(n-i)}
$$

with equality if and only if $K$ is a dilation of $L$.

This is contained in the following theorem.

Theorem 3.7. If $K_{j}(j=1,2, \ldots, m) \in \mathscr{K}^{n}$, then, for $i<n-1$,

$$
\left(\sum_{j=1}^{m} \tilde{W}_{i}\left(K_{j}\right)\right)^{1 /(n-i)} \leq \tilde{W}_{i}\left(K_{1} \tilde{+} \cdots \tilde{+} K_{m}\right)^{1 /(n-i)} \leq \sum_{j=1}^{m} \tilde{W}_{i}\left(K_{j}\right)^{1 /(n-i)} .
$$

The right equality of (3.21) holds if and only if $K_{j}$ are all dilations of each other; the left equality of (3.21) holds if and only if, for almost all $u$, all but one of $\rho\left(K_{j}, u\right)$ are zero.

For $i>n$ or $n-1<i<n$,

$$
\tilde{W}_{i}\left(K_{1} \tilde{+} \cdots \tilde{+} K_{m}\right)^{1 /(n-i)} \geq \sum_{j=1}^{m} \tilde{W}_{i}\left(K_{j}\right)^{1 /(n-i)}
$$

with equality if and only if $K_{j}$ are all dilations of each other.

Proof. We only give the proof of (3.21). From (2.15), (2.19), and in view of the Minkowski inequality for integrals, we obtain

$$
\begin{aligned}
\tilde{W}_{i}\left(K_{1} \tilde{+} \cdots \tilde{+} K_{m}\right)^{1 /(n-i)} & =\left(\frac{1}{n} \int_{S^{n-1}} \rho\left(K_{1} \tilde{+} \cdots \tilde{+} K_{m}, u\right)^{n-i} d S(u)\right)^{1 /(n-i)} \\
& =\left(\frac{1}{n} \int_{S^{n-1}}\left(\sum_{j=1}^{m} \rho\left(K_{j}, u\right)\right)^{n-i} d S(u)\right)^{1 /(n-i)} \\
& \leq \sum_{j=1}^{m}\left(\frac{1}{n} \int_{S^{n-1}} \rho\left(K_{j}, u\right)^{n-i} d S(u)\right)^{1 /(n-i)} \\
& =\sum_{j=1}^{m} \tilde{W}_{i}\left(K_{j}\right)^{1 /(n-i)} .
\end{aligned}
$$

On the other hand, we have

$$
\begin{aligned}
\tilde{W}_{i}\left(K_{1} \tilde{+} \cdots \tilde{+} K_{m}\right) & =\frac{1}{n} \int_{S^{n-1}} \rho\left(\sum_{j=1}^{m} K_{j}, u\right)^{n-i} d S(u) \\
& \geq \sum_{j=1}^{m} \int_{S^{n-1}} \rho\left(K_{j}, u\right)^{n-i} d S(u) \\
& =\sum_{j=1}^{m} \tilde{W}_{i}\left(K_{j}\right) .
\end{aligned}
$$

Inequality (3.21) follows from (3.23) and (3.24). 
906 Some new Brunn-Minkowski-type inequalities in convex bodies

In (3.21), let $i=0$; we have the following corollary.

Corollary 3.8. If $K_{j}(j=1,2, \ldots, m) \in \mathscr{Y}^{n}$, then

$$
\begin{aligned}
\left(V\left(K_{1}\right)+\cdots+V\left(K_{m}\right)\right)^{1 / n} & \leq V\left(K_{1} \tilde{+} \cdots \tilde{+} K_{m}\right)^{1 / n} \\
& \leq V\left(K_{1}\right)^{1 / n}+\cdots+V\left(K_{m}\right)^{1 / n}
\end{aligned}
$$

The right equality of (3.25) holds if and only if $K_{j}$ are all dilations of each other; the left equality of (3.25) holds if and only if, for almost all $u$, all but one of $\rho\left(K_{j}, u\right)$ are zero.

In (3.22), let $i=2 n$ to obtain the following corollary.

Corollary 3.9. If $K_{j}(j=1,2, \ldots, m) \in \mathscr{Y}^{n}$, then

$$
\begin{aligned}
\left(\tilde{W}_{2 n}\left(K_{1}\right)+\cdots+\tilde{W}_{2 n}\left(K_{m}\right)\right)^{-1 / n} & \leq \tilde{W}_{2 n}\left(K_{1} \tilde{+} \cdots \tilde{+} K_{m}\right)^{-1 / n} \\
& \leq \tilde{W}_{2 n}\left(K_{1}\right)^{-1 / n}+\cdots+\tilde{W}_{2 n}\left(K_{m}\right)^{-1 / n} .
\end{aligned}
$$

The right equality of (3.26) holds if and only if $K_{j}$ are all dilations of each other; the left equality of (3.26) holds if and only if, for almost all $u$, all but one of $\rho\left(K_{j}, u\right)$ are zero.

The dual Minkowski inequality (3.17) is contained in the following theorem.

Theorem 3.10. Let $K, L \in \mathcal{Y}^{n}, i \in \mathbb{R}$; if $0<i<n$,

$$
\tilde{V}_{i}(K, L)^{n} \leq V(K)^{n-i} V(L)^{i}
$$

with equality if and only if $K$ is a dilation of $L$, while if $i>n$ or $i<0$,

$$
\tilde{V}_{i}(K, L)^{n} \geq V(K)^{n-i} V(L)^{i}
$$

with equality if and only if $K$ is a dilation of $L$.

To prove (3.27), we use (2.18) and in view of the Hölder inequality for integrals (see Hardy et al. [11], page 140) with $p=n /(n-i), q=n / i, f(u)=\rho(K, u)^{n-i}, g(u)=\rho(L, u)^{i}$. Similarly, we also can prove (3.28).

An immediate consequence of Theorem 3.10 is the following theorem.

Theorem 3.11. If $\gamma \subset \varphi^{n}, K, L \in \varphi^{n}$, then $i \neq 0$ and $i \neq n$ if either

$$
\tilde{V}_{i}(K, M)=\tilde{V}_{i}(L, M), \quad M \in \gamma,
$$

or

$$
\tilde{V}_{i}(M, K)=\tilde{V}_{i}(M, L), \quad M \in \gamma,
$$

holds, it follows that $K=L$. 
Proof. We only prove the case of $i>n$. Suppose (3.29) holds. Take $K$ for $M$, use (3.28), and in view of (2.9) and (2.17), we get

$$
\begin{gathered}
V(K)^{n}=\tilde{V}(K)^{n}=\tilde{V}_{i}(L, K)^{n} \geq V(L)^{n-i} V(K)^{i}, \\
V(K) \leq V(L)
\end{gathered}
$$

with equality if and only if $K$ and $L$ are dilations. Now take $L$ for $M$ and get

$$
V(L) \leq V(K)
$$

Since $V(K)=V(L)$ and $K$ and $L$ are dilations, the proof is complete. The same sort of argument shows that (3.30) leads to the same conclusion.

Remark 3.12. When $n>i>0$ and let $i=1$, Theorem 3.11 reduces to the following result.

If $\gamma \subset \varphi^{n}, K, L \in \varphi^{n}$, then if either

$$
\tilde{V}_{1}(K, M)=\tilde{V}_{1}(L, M), \quad M \in \gamma,
$$

or

$$
\tilde{V}_{1}(M, K)=\tilde{V}_{1}(M, L), \quad M \in \gamma,
$$

holds, it follows that $K=L$.

This is also a new result which was given by Lutwak [17].

\subsection{The dual Brunn-Minkowski inequality for the radial Blaschke linear combination.} Lutwak [17] proved the following result.

If $K, L \in \varphi^{n}$, then

$$
V(K \breve{+} L)^{(n-1) / n} \leq V(K)^{(n-1) / n}+V(L)^{(n-1) / n}
$$

with equality if and only if $K$ is a dilation of $L$.

Our main result is given in the following theorem.

Theorem 3.13. If $K, L \in \varphi^{n}, i \in \mathbb{R}$, then, for $i<1$,

$$
\tilde{W}_{i}(K \breve{+} L)^{(n-1) /(n-i)} \leq W_{i}(K)^{(n-1) /(n-i)}+W_{i}(L)^{(n-1) /(n-i)},
$$

with equality if and only if $K$ and $L$ are dilations, while for $n>i>1$ or $i>n$,

$$
\tilde{W}_{i}(K \breve{+} L)^{(n-1) /(n-i)} \geq W_{i}(K)^{(n-1) /(n-i)}+W_{i}(L)^{(n-1) /(n-i)},
$$

with equality if and only if $K$ and $L$ are dilations. 
908 Some new Brunn-Minkowski-type inequalities in convex bodies

Proof. We only give the proof of (3.36). From (2.19), (2.20), and in view of the Minkowski inequality for integrals, we obtain

$$
\begin{aligned}
\tilde{W}_{i}(K \breve{+} L)^{(n-1) /(n-i)} \\
\quad=\left(\frac{1}{n} \int_{S^{n-1}} \rho(K \breve{+} L, u)^{n-i} d S(u)\right)^{(n-1) /(n-i)} \\
\quad=\left(\frac{1}{n} \int_{S^{n-1}}\left(\rho(K, u)^{n-1}+\rho(L, u)^{n-1}\right)^{(n-i) /(n-1)} d S(u)\right)^{(n-1) /(n-i)} \\
\quad \leq\left(\frac{1}{n} \int_{S^{n-1}} \rho(K, u)^{n-i} d S(u)\right)^{(n-1) /(n-i)}+\left(\frac{1}{n} \int_{S^{n-1}} \rho(L, u)^{n-i} d S(u)\right)^{(n-1) /(n-i)} \\
\quad=W_{i}(K)^{(n-1) /(n-i)}+W_{i}(L)^{(n-1) /(n-i)} .
\end{aligned}
$$

Remark 3.14. If $i=0$, inequality (3.36) reduces to inequality (3.35).

\subsection{The dual Brunn-Minkowski inequality for the harmonic Blaschke linear combina-} tion. Lutwak [18] has proved the following important result.

If $K, L \in \varphi^{n}$, and $\lambda, \mu>0$, then

$$
V(\lambda K \hat{+} \mu L)^{1 / n} \geq \lambda V(K)^{1 / n}+\mu V(L)^{1 / n}
$$

with equality if and only if $K$ and $L$ are dilations.

Our main result is given in the following theorem.

Theorem 3.15. $K, L \in \varphi^{n}, i \in \mathbb{R}$, then, for $i<-1$,

$$
\frac{\tilde{W}_{i}(K \hat{+} L)^{(n+1) /(n-i)}}{V(K \hat{+} L)} \leq \frac{\tilde{W}_{i}(K)^{(n+1) /(n-i)}}{V(K)}+\frac{\tilde{W}_{i}(L)^{(n+1) /(n-i)}}{V(L)}
$$

with equality if and only if $K$ and $L$ are dilations, while, for $n>i>-1$ or $i>n$,

$$
\frac{\tilde{W}_{i}(K \hat{+} L)^{(n+1) /(n-i)}}{V(K \hat{+} L)} \geq \frac{\tilde{W}_{i}(K)^{(n+1) /(n-i)}}{V(K)}+\frac{\tilde{W}_{i}(L)^{(n+1) /(n-i)}}{V(L)}
$$

with equality if and only if $K$ and $L$ are dilations. 
Proof. We only give the proof of (3.40). From (2.19), (2.22), (2.23), and in view of the Minkowski inequality for integrals, we have

$$
\begin{aligned}
& \tilde{W}_{i}(K \hat{+} L)^{(n+1) /(n-i)} \\
&=\left(\frac{1}{n} \int_{S^{n-1}} \rho(K \hat{+} L, u)^{n-i} d S(u)\right)^{(n+1) /(n-i)} \\
&=\left(\frac{1}{n} \int_{S^{n-1}}\left(\left(\xi V(K)^{-1} \rho(K, u)^{n+1}+\xi V(L)^{-1} \rho(L, u)^{n+1}\right)^{1 /(n+1)}\right)^{n-i} d S(u)\right)^{(n+1) /(n-i)} \\
& \leq\left(\frac{1}{n} \int_{S^{n-1}}\left(\xi V(K)^{-1} \rho(K, u)^{n+1}\right)^{(n-i) /(n+1)} d S(u)\right)^{(n+1) /(n-i)} \\
&+\left(\frac{1}{n} \int_{S^{n-1}}\left(\xi V(L)^{-1} \rho(L, u)^{n+1}\right)^{(n-i) /(n+1)} d S(u)\right)^{(n+1) /(n-i)} \\
&= \xi V(K)^{-1} W_{i}(K)^{(n+1) /(n-i)}+\xi V(L)^{-1} W_{i}(L)^{(n+1) /(n-i)} .
\end{aligned}
$$

Remark 3.16. When $i=0$, inequality (3.41) reduces to the following:

$$
V(K \hat{+} L)^{1 / n} \geq V(K)^{1 / n}+V(L)^{1 / n}
$$

with equality if and only if $K$ and $L$ are dilations.

This is a new inequality similar to inequality (3.39).

3.5. The Minkowski inequality and Brunn-Minkowski inequalities of affine surface area for the Blaschke linear combination. The following inequality was established by Lutwak [16]. If $K, L \in \kappa^{n}$, and $i \in \mathbb{R}$, then for $i<-1$,

$$
\Omega_{i}(K \ddot{+} L)^{(n+1) /(n-i)} \leq \Omega_{i}(K)^{(n+1) /(n-i)}+\Omega_{i}(L)^{(n+1) /(n-i)}
$$

with equality if and only if $K$ and $L$ are homothetic, while, for $i>-1$,

$$
\Omega_{i}(K \ddot{+} L)^{(n+1) /(n-i)} \geq \Omega_{i}(K)^{(n+1) /(n-i)}+\Omega_{i}(L)^{(n+1) /(n-i)}
$$

with equality if and only if $K$ and $L$ are homothetic.

These two inequalities are just the special cases of the following theorem.

Theorem 3.17. If $K_{j}(j=1, \ldots, m) \in \kappa^{n}$, then, for $i<-1$,

$$
\left(\sum_{j=1}^{m} \Omega_{i}\left(K_{j}\right)\right)^{(n+1) /(n-i)} \leq \Omega_{i}\left(K_{1} \ddot{+} \cdots \ddot{+} K_{m}\right)^{(n+1) /(n-i)} \leq \sum_{j=1}^{m}\left(\Omega_{i}\left(K_{j}\right)^{(n+1) /(n-i)}\right) .
$$


910 Some new Brunn-Minkowski-type inequalities in convex bodies

The right equality of (3.46) holds if and only if $K_{j}$ are homothetic; the left equality of (3.46) holds if and only if, for almost all $u$, all but one of $\rho\left(K_{j}, u\right)$ are zero, while, for $-1<i<n$ or $i>n$,

$$
\Omega_{i}\left(K_{1} \ddot{+} \cdots \ddot{+} K_{m}\right)^{(n+1) /(n-i)} \geq \sum_{j=1}^{m}\left(\Omega_{i}\left(K_{j}\right)^{(n+1) /(n-i)}\right)
$$

with equality if and only if $K_{j}$ are homothetic.

To prove (3.46), we use (2.27), (2.31), (2.33), and in view of the Minkowski inequality with $p=(n-i) /(n+1), f_{j}(u)=f\left(\Pi K_{j}, u\right)$. Similarly, we also can prove (3.47).

A general version of the dual Minkowski inequality for the affine surface area will be given in the following theorem.

Theorem 3.18. Let $K, L \in \kappa^{n}, i \in \mathbb{R}$; if $n>i>0$,

$$
\Omega_{i}(K, L)^{n} \leq \Omega(K)^{n-i} \Omega(L)^{i},
$$

while, if $i<0$ or $i>n$,

$$
\Omega_{i}(K, L)^{n} \geq \Omega(K)^{n-i} \Omega(L)^{i}
$$

with equality if and only if $K$ and $L$ are homothetic.

To prove (3.48), we use the Hölder inequality for integrals with $p=n /(n-i), q=n / i$, $f(u)=f(K, u)^{(n-i) /(n+1)}, g(u)=f(L, u)^{i /(n+1)}$, and (2.30). Similarly, we also can prove (3.49).

An immediate consequence of Theorem 3.18 is the following theorem.

Theorem 3.19. If $\gamma \subset \kappa^{n}, K, L, M \in \gamma$, then $i \neq 0$ and $i \neq n$ if either

$$
\Omega_{i}(K, M)=\Omega_{i}(L, M),
$$

or

$$
\Omega_{i}(M, K)=\Omega_{i}(M, L),
$$

then, $K$ and $L$ are translates.

Proof. We only prove the case of $i<0$. Suppose (3.50) holds. Take $K$ for $M$, use (3.49), to get

$$
\Omega(K) \leq \Omega(L)
$$

with equality if and only if $K$ and $L$ are homothetic. Now take $L$ for $M$ and get

$$
\Omega(L) \leq \Omega(K) .
$$


Since $\Omega(K)=\Omega(L)$ and $K$ and $L$ are homothetic, $K$ and $L$ must be translates. The same sort of argument shows that (3.51) leads to the same conclusion.

In Theorem 3.19, when $i=-1$; we have the following corollary.

Corollary 3.20. If $\gamma \subset \kappa^{n}, K, L, M \in \gamma$, then, either

$$
\Omega_{-1}(K, M)=\Omega_{-1}(L, M)
$$

or

$$
\Omega_{-1}(M, K)=\Omega_{-1}(M, L)
$$

then $K$ and $L$ are translates.

This is just a new result which was also given by Lutwak [18].

Some new inequalities of the affine surface area for the harmonic Blaschke linear combination will be established.

If $K, L \in \varphi_{c}^{n}$, for $i<-1$,

$$
\Omega_{i}(\Lambda(K \hat{+} L))^{(n+1) /(n-i)} \leq \Omega_{i}(\Lambda K)^{(n+1) /(n-i)}+\Omega_{i}(\Lambda L)^{(n+1) /(n-i)}
$$

with equality if and only if $K$ and $L$ are homothetic.

For $i>-1$ or $i>n$,

$$
\Omega_{i}(\Lambda(K \hat{+} L))^{(n+1) /(n-i)} \geq \Omega_{i}(\Lambda K)^{(n+1) /(n-i)}+\Omega_{i}(\Lambda L)^{(n+1) /(n-i)}
$$

with equality if and only if $K$ and $L$ are homothetic.

In (3.57), let $i=0$ to obtain

$$
\Omega(\Lambda(K \hat{+} L))^{(n+1) / n} \geq \Omega(\Lambda K)^{(n+1) / n}+\Omega(\Lambda L)^{(n+1) / n}
$$

with equality if and only if $K$ and $L$ are homothetic.

These inequalities are just the special cases of the following theorem.

Theorem 3.21. If $K_{j}(j=1, \ldots, m) \in \varphi_{c}^{n}$, then, for $i<-1$,

$$
\left(\sum_{j=1}^{m} \Omega_{i}\left(\Lambda K_{j}\right)\right)^{(n+1) /(n-i)} \leq \Omega_{i}\left(\Lambda\left(K_{1} \hat{+} \cdots \hat{+} K_{m}\right)\right)^{(n+1) /(n-i)} \leq \sum_{j=1}^{m}\left(\Omega_{i}\left(\Lambda K_{j}\right)^{(n+1) /(n-i)}\right) .
$$

The right equality of (3.59) holds if and only if $K_{j}$ are homothetic; the left equality of (3.59) holds if and only if, for almost all $u$, all but one of $f\left(\Lambda K_{j}, u\right)$ are zero. 
912 Some new Brunn-Minkowski-type inequalities in convex bodies

While, for $i>n$ or $i>-1$,

$$
\Omega_{i}\left(\Lambda\left(K_{1} \hat{+} \cdots \hat{+} K_{m}\right)\right)^{(n+1) /(n-i)} \geq \sum_{j=1}^{m}\left(\Omega_{i}\left(\Lambda K_{j}\right)^{(n+1) /(n-i)}\right)
$$

with equality if and only if $K_{j}$ are homothetic.

Proof. We only give the proof of (3.59). From (2.27), (2.28), (2.31), (2.33), and the Minkowski inequality, we obtain

$$
\begin{aligned}
\Omega_{i}(\Lambda & \left.\left(K_{1} \hat{+} \cdots \hat{+} K_{m}\right)\right)^{(n+1) /(n-i)} \\
& =\left(\frac{1}{n} \int_{S^{n-1}} f\left(\Lambda\left(K_{1} \hat{+} \cdots \hat{+} K_{m}\right), u\right)^{(n-i) /(n+1)} d S(u)\right)^{(n+1) /(n-i)} \\
& =\left(\frac{1}{n} \int_{S^{n-1}} f\left(\left(\Lambda K_{1} \ddot{+} \cdots \ddot{+} \Lambda K_{m}\right), u\right)^{(n-i) /(n+1)} d S(u)\right)^{(n+1) /(n-i)} \\
& =\left(\frac{1}{n} \int_{S^{n-1}}\left(\sum_{j=1}^{m} f\left(\Lambda K_{j}, u\right)\right)^{(n-i) /(n+1)} d S(u)\right)^{(n+1) /(n-i)} \\
& \leq \sum_{j=1}^{m}\left(\frac{1}{n} \int_{S^{n-1}} f\left(\Lambda K_{j}, u\right)^{(n-i) /(n+1)} d S(u)\right)^{(n+1) /(n-i)} \\
& =\sum_{j=1}^{m} \Omega_{i}\left(\Lambda K_{j}\right)^{(n+1) /(n-i)} .
\end{aligned}
$$

On the other hand, we have

$$
\begin{aligned}
\Omega_{i}\left(\Lambda\left(K_{1} \hat{+} \cdots \hat{+} K_{m}\right)\right) & =\frac{1}{n} \int_{S^{n-1}}\left(\sum_{i=1}^{m} f\left(\Lambda K_{j}, u\right)\right)^{(n+1) /(n-i)} d S(u) \\
& \geq \sum_{j=1}^{m} \int_{S^{n-1}} f\left(\Lambda K_{j}, u\right)^{(n+1) /(n-i)} d S(u) \\
& =\sum_{j=1}^{m} \Omega_{i}\left(\Lambda K_{j}\right) .
\end{aligned}
$$

From (3.61) and (3.62), we get (3.59).

In (3.60), let $i=0$; we have the following corollary.

Corollary 3.22. If $K_{j}(j=1, \ldots, m) \in \varphi_{c}^{n}$, then

$$
\Omega\left(\Lambda\left(K_{1} \hat{+} \cdots \hat{+} K_{m}\right)\right)^{(n+1) / n} \geq \Omega\left(\Lambda K_{1}\right)^{(n+1) / n}+\cdots+\Omega\left(\Lambda K_{m}\right)^{(n+1) / n}
$$

with equality if and only if $K_{j}$ are homothetic.

In (3.60), let $i=2 n$; we have the following corollary. 
Corollary 3.23. If $K_{j}(j=1, \ldots, m) \in \varphi_{c}^{n}$, then

$$
\Omega_{2 n}\left(\Lambda\left(K_{1} \hat{+} \cdots \hat{+} K_{m}\right)\right)^{-(n+1) / n} \geq \Omega_{2 n}\left(\Lambda K_{1}\right)^{-(n+1) / n}+\cdots+\Omega_{2 n}\left(\Lambda K_{m}\right)^{-(n+1) / n}
$$

with equality if and only if $K_{j}$ are homothetic.

3.6. The dual Brunn-Minkowski inequality of the affine surface area for the Lutwak linear combination. Lutwak [18] proved the following result.

If $K, L \in v^{n}$ and $\lambda, \mu \geq 0$, then

$$
\Omega(\lambda \circ K \check{+} \mu \circ L)^{-1 / n} \geq \lambda \Omega(K)^{-1 / n}+\mu \Omega(L)^{-1 / n}
$$

with equality if and only if $K$ and $L$ are homothetic.

Our main result is given in the following theorem.

Theorem 3.24. If $K, L \in v^{n}, i \in \mathbb{R}$, then, for $i<n$ or $n<i<n+1$,

$$
\Omega_{i}(K \check{+} L)^{-1 /(n-i)} \geq \Omega_{i}(K)^{-1 /(n-i)}+\Omega_{i}(L)^{-1 /(n-i)},
$$

while, for $i>n+1$,

$$
\Omega_{i}(K \check{+} L)^{-1 /(n-i)} \leq \Omega_{i}(K)^{-1 /(n-i)}+\Omega_{i}(L)^{-1 /(n-i)}
$$

with equality if and only if $K$ and $L$ are homothetic.

Proof. We only give the proof of (3.66).

From (2.31), (2.37), and in view of the Minkowski inequality, we obtain

$$
\begin{aligned}
\Omega_{i}(K \check{+} & L)^{-1 /(n-i)} \\
= & \left(\frac{1}{n} \int_{S^{n-1}} f(K \check{+} L, u)^{(n-i) /(n+1)} d S(u)\right)^{-1 /(n-i)} \\
= & \left(\frac{1}{n} \int_{S^{n-1}}\left(\left(f(K, u)^{-1 /(n+1)}+f(L, u)^{-1 /(n+1)}\right)^{-(n+1)}\right)^{(n-i) /(n+1)} d S(u)\right)^{-1 /(n-i)} \\
\geq & \left(\frac{1}{n} \int_{S^{n-1}} f(K, u)^{(n-i) /(n+1)} d S(u)\right)^{-1 /(n-i)} \\
& +\left(\frac{1}{n} \int_{S^{n-1}} f(L, u)^{(n-i) /(n+1)} d S(u)\right)^{-1 /(n-i)} \\
= & \Omega_{i}(K)^{-1 /(n-i)}+\Omega_{i}(L)^{-1 /(n-i)} .
\end{aligned}
$$


914 Some new Brunn-Minkowski-type inequalities in convex bodies

Remark 3.25. In (3.66), let $i=0$, inequality (3.66) reduces to the following:

$$
\Omega(K \check{+} L)^{-1 / n} \geq \Omega(K)^{-1 / n}+\Omega(L)^{-1 / n},
$$

with equality if and only if $K$ and $L$ are homothetic.

Here, we call (3.69) to be the Minkowski inequality for the Lutwak linear combination. This is just a new inequality similar to inequality (3.65).

\section{Acknowledgments}

This work was supported by the National Natural Sciences Foundation of China (10271071). It was also supported by the academic mainstay of Middle Age and Youth Foundation of Shandong Province of China.

\section{References}

[1] K. Ball, Volumes of sections of cubes and related problems, Geometric Aspects of Functional Analysis (1987-88), Lecture Notes in Math., vol. 1376, Springer, Berlin, 1989, pp. 251-260.

[2] Shadows of convex bodies, Trans. Amer. Math. Soc. 327 (1991), no. 2, 891-901.

[3] - Volume ratios and a reverse isoperimetric inequality, J. London Math. Soc. (2) 44 (1991), no. 2, 351-359.

[4] W. Blaschke, Vorlesungen über Integralgeometrie. I, Chelsea Publishing, New York, 1949.

[5]_ Vorlesungen über Integralgeometrie. II, Chelsea Publishing, New York, 1949.

[6] T. Bonnesen and W. Fenchel, Theorie der konvexen Körper, Springer, Berlin, 1934.

[7] J. Bourgain and J. Lindenstrauss, Projection bodies, Geometric Aspects of Functional Analysis (1986-87), Lecture Notes in Math., vol. 1317, Springer, Berlin, 1988, pp. 250-270.

[8] R. J. Gardner, Intersection bodies and the Busemann-Petty problem, Trans. Amer. Math. Soc. 342 (1994), no. 1, 435-445.

[9] - A positive answer to the Busemann-Petty problem in three dimensions, Ann. of Math. (2) 140 (1994), no. 2, 435-447.

[10] Geometric Tomography, Encyclopedia Math. Appl., vol. 58, Cambridge University Press, Cambridge, 1995.

[11] G. H. Hardy, J. E. Littlewood, and G. Pólya, Inequalities, Cambridge University Press, Cambridge, 1934.

[12] E. Lutwak, Dual mixed volumes, Pacific J. Math. 58 (1975), no. 2, 531-538.

[13] Width-integrals of convex bodies, Proc. Amer. Math. Soc. 53 (1975), no. 2, 435-439.

[14] Mixed projection inequalities, Trans. Amer. Math. Soc. 287 (1985), no. 1, 91-105.

[15] _ Volume of mixed bodies, Trans. Amer. Math. Soc. 294 (1986), no. 2, 487-500.

[16] _ Mixed affine surface area, J. Math. Anal. Appl. 125 (1987), no. 2, 351-360.

[17] Intersection bodies and dual mixed volumes, Adv. Math. 71 (1988), no. 2, 232-261.

[18] Centroid bodies and dual mixed volumes, Proc. London Math. Soc. (3) 60 (1990), no. 2, 365-391.

[19] On quermassintegrals of mixed projection bodies, Geom. Dedicata 33 (1990), no. 1, $51-58$.

[20] Extended affine surface area, Adv. Math. 85 (1991), no. 1, 39-68.

[21] Inequalities for mixed projection bodies, Trans. Amer. Math. Soc. 339 (1993), no. 2, 901-916.

[22] , The Brunn-Minkowski-Firey theory. I. Mixed volumes and the Minkowski problem, J. Differential Geom. 38 (1993), no. 1, 131-150. 
[23] - The Brunn-Minkowski-Firey theory. II. Affine and geominimal surface areas, Adv. Math. 118 (1996), no. 2, 244-294.

[24] C. M. Petty, Geominimal surface area, Geom. Dedicata 3 (1974), 77-97.

[25] R. Schneider, Convex Bodies: The Brunn-Minkowski Theory, Encyclopedia Math. Appl., vol. 44, Cambridge University Press, Cambridge, 1993.

Zhao Chang-Jian: Department of Applied Mathematics, College of Sciences, China Jiliang University, Hangzhou 310014, China

E-mail address: chjzhao@163.com

Leng Gangsong: Department of Mathematics, College of Sciences, Shanghai University, Shanghai 200436, China

Lokenath Debnath: Department of Mathematics, University of Texas - Pan American, Edinburg, TX 78539-2999, USA

E-mail address: debnathl@panam.edu 


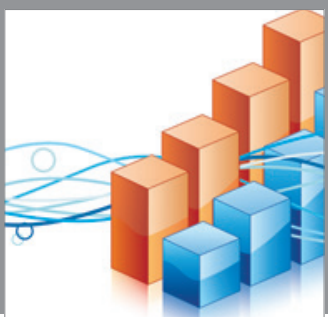

Advances in

Operations Research

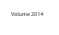

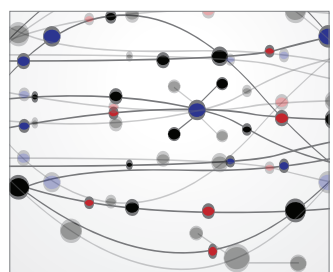

\section{The Scientific} World Journal
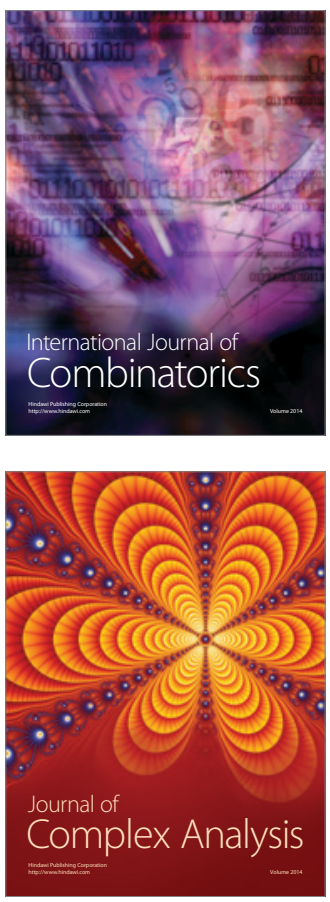

International Journal of

Mathematics and

Mathematical

Sciences
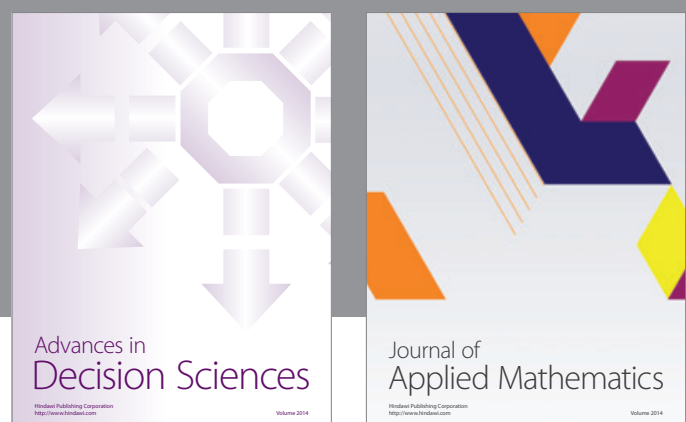

Journal of

Applied Mathematics
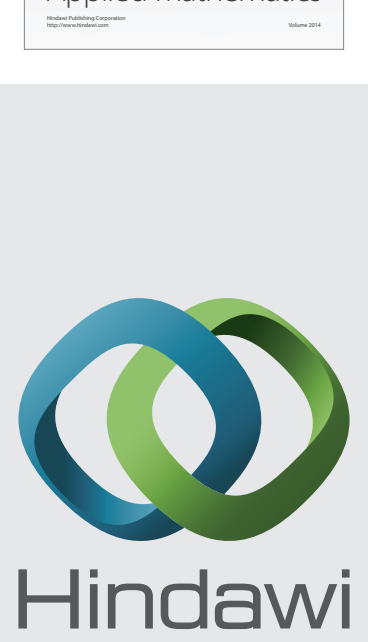

Submit your manuscripts at http://www.hindawi.com
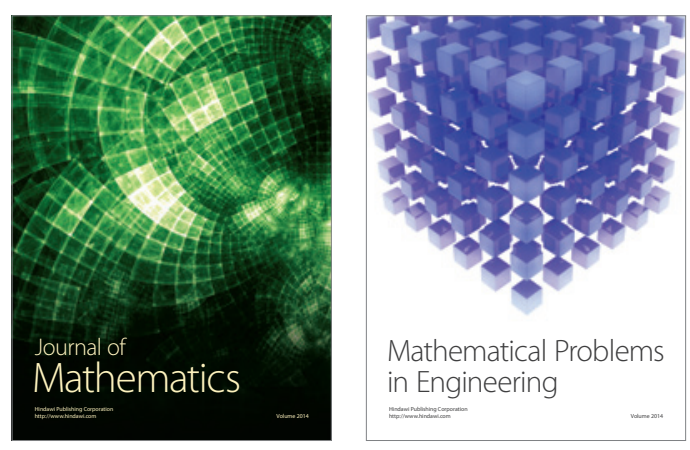

Mathematical Problems in Engineering
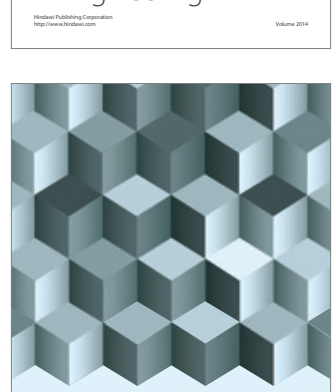

Journal of

Function Spaces
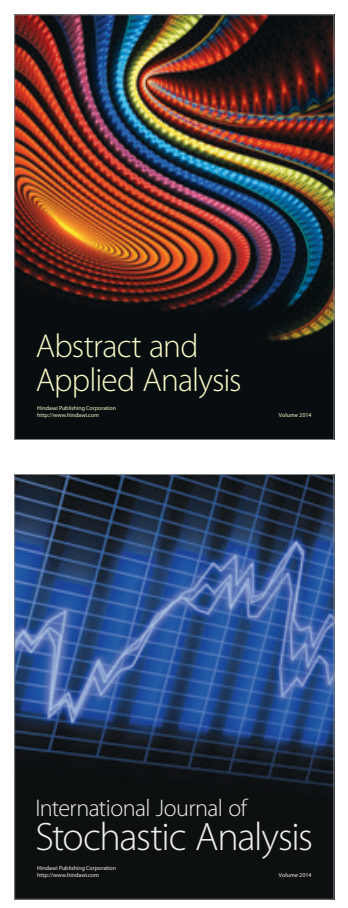

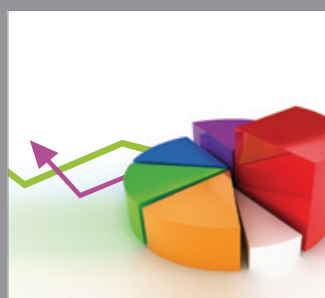

ournal of

Probability and Statistics

Promensencen
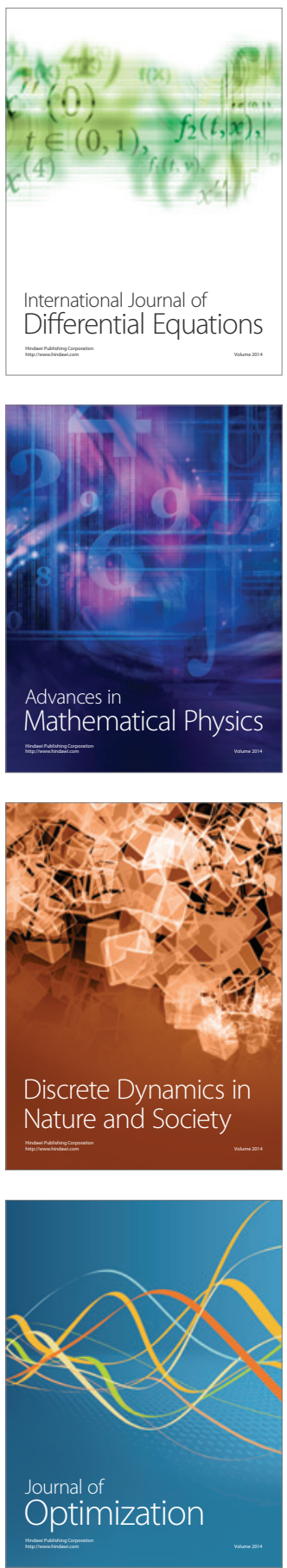